\title{
EXISTENCE AND UNIQUENESS OF GLOBAL SOLUTIONS FOR THE MODIFIED ANISOTROPIC 3D NAVIER-STOKES EQUATIONS
}

\author{
HAKIMA BESSAIH, SABER TRABELSI, AND HAMDI ZORGATI
}

\begin{abstract}
We study a modified three-dimensional incompressible anisotropic Navier-Stokes equations. The modification consists in the addition of a power term to the nonlinear convective one. This modification appears naturally in porous media when a fluid obeys the Darcy-Forchheimer law instead of the classical Darcy law. We prove global in time existence and uniqueness of solutions without assuming the smallness condition on the initial data. This improves the result obtained for the classical $3 D$ incompressible anisotropic Navier-Stokes equations.
\end{abstract}

MSC Subject Classifications: 35Q30, 35Q35, 76D05, 76D03, 76S05.

Keywords: Navier-Stokes equations, Brinkman-Forchheimer-extended Darcy model, anisotropic viscosity.

\section{INTRODUCTION}

The purpose of this paper is to study the following modified Navier-Stokes system

$$
\mathcal{S}_{a}:\left\{\begin{aligned}
\partial_{t} u-\nu \Delta_{h} u+(u \cdot \nabla) u+a|u|^{2 \alpha} u & =-\nabla p & & \text { for } \quad(t, x) \in \mathbb{R}^{3} \times \mathbb{R}^{3}, \\
\nabla \cdot u & =0 & & \text { for } \quad(t, x) \in \mathbb{R}^{3} \times \mathbb{R}^{3}, \\
u_{\left.\right|_{t=0}} & =u_{0}, & &
\end{aligned}\right.
$$

where $\partial_{t}$ denotes the partial derivative with respect to time, $\alpha \in \mathbb{R}, a>0, \Delta_{h}:=$ $\partial_{1}^{2}+\partial_{2}^{2}$ and $\partial_{i}$ denotes the partial derivative in the direction $x_{i}$. Clearly, $\mathcal{S}_{0}$ corresponds to the classical anisotropic Navier-Stokes equations. When a Coriolis force $\frac{1}{\epsilon} u \times \mathbf{e}_{3}$ is added, where $\mathbf{e}_{3}$ denotes the unit vertical vector and $\epsilon>0$ is the so called Rossby number, system $\mathcal{S}_{0}$ models rotating flows (see e.g. [12]). We refer the reader to e.g. [6] and [2] where the relevance of considering anisotropic viscosities of the form $\nu_{h} \Delta_{h} u+\epsilon \nu_{v} \partial_{3}^{2} u$ is explained through the Ekman's law. For a complete discussion leading to the anisotropic Navier-Stokes systems, the reader is referred to the book [12] or the introduction of the book [5]. System $\mathcal{S}_{a}$ with $\Delta_{h}$ replaced by the classical Laplacian is nothing but the three-dimensional BrinkmanForchheimer-extended Darcy model. The Brinkman-Forchheimer-extended Darcy equations

$$
\partial_{t} u-\nu \Delta u+(u \cdot \nabla) u+\nabla p+a|u|^{2 \alpha} u=f, \quad \nabla \cdot u=0,
$$

have been extensively studied. The existence of weak solutions for $\alpha \geq 0$ and existence (for $\alpha \geq \frac{5}{4}$ ) and uniqueness (for $\frac{5}{4}<\alpha \leq 2$ ) of strong solutions of (11) is shown in [3]. Also, in [10, existence and uniqueness of weak and strong solutions is shown for a larger range of $\alpha$. In [7, the authors show existence and 
uniqueness of solutions for all $\alpha>1$, with Dirichlet boundary conditions and regular enough initial data. Their argument relies on the maximal regularity estimate for the corresponding semi-linear stationary Stokes problem proved using some modification of the nonlinear localization technique. In [10, the authors showed the existence and uniqueness of weak and strong solutions, in particular with initial data in $H^{1}$ instead of $H^{2}$ in $[7$ with periodic boundary conditions. Let us mention that the space $L^{2 \alpha+2}$ appears naturally in the mathematical analysis of system (1), and it coincides obviously with $L^{4}$ for $\alpha=1$. Since $\dot{H}^{\frac{1}{2}}$ is known to be the critical Sobolev space for the classical 3D Navier-Stokes equations and $\dot{H}^{\frac{1}{2}} \subset L^{4}$, then it makes sense to assume $\alpha>1$. For a more detailed discussion about equation (1) and the various values of $\alpha$ that lead to the well posedness, we refer the reader to [7, 10] and the references therein.

Before going further, let us precise the notation and the functional setting that will be used along the paper. Since the horizontal variable $x_{h} \delta\left(x_{1}, x_{2}\right)$ does not play the same role as the vertical variable $x_{3}$, it is natural to introduce functional spaces taking into account this feature. These spaces are the so called anisotropic Sobolev spaces $H^{s, s^{\prime}}$ for all $s, s^{\prime} \in \mathbb{R}$. More precisely, the space $H^{s, s^{\prime}}$ is the Sobolev space with regularity $H^{s}$ in $x_{h}$ and $H^{s^{\prime}}$ in $x_{3}$. Let $\langle x\rangle$ denote the quantity $\langle x\rangle \delta\left(1+|x|^{2}\right)^{\frac{1}{2}}$, then for all $s, s^{\prime} \in \mathbb{R}, H^{s, s^{\prime}}$ is the space of tempered distributions $\psi \in \mathcal{S}^{\prime}\left(\mathbb{R}^{3}\right)$ which satisfy

$$
\|\psi\|_{s, s^{\prime}} \delta \int_{\Omega}\left\langle\xi^{\prime}\right\rangle^{2 s}\left\langle\xi_{3}\right\rangle^{2 s^{\prime}}|\mathscr{F} \psi(\xi)|^{2} d \xi
$$

where $\xi^{\prime}:=\left(\xi_{1}, \xi_{2}\right)$ and $\mathscr{F}$ denotes the Fourier transform. The space $\|\psi\|_{H^{s, s^{\prime}}}$ endowed with the norm $\|\cdot\|_{s, s^{\prime}}$ is a Hilbert space. Obviously, the homogenous anisotropic Sobolev space $\dot{H}^{s, s^{\prime}}\left(\mathbb{R}^{3}\right)$ are obtained by replacing $\langle\cdot\rangle$ by $|\cdot|$. We will denote $L_{h}^{p}\left(L_{v}^{q}\right)$ the space $L^{p}\left(\mathbb{R}_{x_{1}} \times \mathbb{R}_{x_{2}} ; L^{q}\left(\mathbb{R}_{x_{3}}\right)\right)$ endowed with the norm

$\|\psi\|_{L_{h}^{p}\left(L_{v}^{q}\right)} \delta\|\| \psi\left\|_{L^{q}\left(\mathbb{R}_{x_{3}}\right)}\right\|_{L^{p}\left(\mathbb{R}_{x_{1}} \times \mathbb{R}_{x_{2}}\right)}=\left\{\int_{\mathbb{R}_{x_{1}} \times \mathbb{R}_{x_{2}}}\left(\int_{\mathbb{R}_{x_{3}}}\left|\psi\left(x_{h}, x_{3}\right)\right|^{q} d x_{3}\right)^{\frac{p}{q}} d x_{h}\right\}^{\frac{1}{p}}$.

Equivalently, we denote $L_{v}^{q}\left(L_{h}^{p}\right)$ the space $L^{q}\left(\mathbb{R}_{x_{3}}\left(L^{q}\left(\mathbb{R}_{x_{1}} \times \mathbb{R}_{x_{2}}\right)\right)\right)$ with the associated norm given by $\|\psi\|_{L_{v}^{q}\left(L_{h}^{p}\right)}:=\|\| \psi\left(\cdot, x_{3}\right)\left\|_{L^{p}\left(\mathbb{R}_{x_{1}} \times \mathbb{R}_{x_{2}}\right)}\right\|_{L^{q}\left(\mathbb{R}_{x_{3}}\right)}$. The $L^{p}\left(\mathbb{R}^{3}\right)$ norms will be denoted $\|\cdot\|_{p}$.

The mathematical analysis of the anisotropic Navier-Stokes system $\mathcal{S}_{0}$ was originally investigated in 4 and 8 where it is proved that the system $\mathcal{S}_{0}$ is locally well-posed for initial data in $H^{0, s}\left(\mathbb{R}^{3}\right)$ for all $s>\frac{1}{2}$. Moreover, it has also been proved that if the initial data $u_{0}$ is such that

$$
\left\|u_{0}\right\|_{L^{2}\left(\mathbb{R}^{3}\right)}^{s-\frac{1}{2}}\left\|u_{0}\right\|_{\dot{H}^{0, s}\left(\mathbb{R}^{3}\right)}^{\frac{3}{2}-s} \leq c,
$$

for a sufficiently small constant $c$, then system $\mathcal{S}_{0}$ is globally well-posed. The aim of this short paper is to show how the damping term $|u|^{2 \alpha} u$ gives rise to a smoothing effect in the vertical velocity. Therefore it allows to get rid of the smallness assumption (above) used in $\mathcal{S}_{0}$. Even though, this result is still valid in the critical Sobolev and Besov spaces $H^{0, \frac{1}{2}}$ and $B^{0, \frac{1}{2}}$ (see [11), in order to avoid technicalities, in this paper we chose to focus on a less optimal space to show how we take advantage of the damping term. A similar result in the spaces $H^{0, \frac{1}{2}}$ and 
$B^{0, \frac{1}{2}}$ will be shown in a forthcoming paper soon. Here, we specifically show the following

Theorem 0.1. Let $a, \nu>0, \alpha>1$ and $u_{0} \in H^{0,1}\left(\mathbb{R}^{3}\right)$ such that $\operatorname{div} u_{0}=0$. Then, system $\mathcal{S}_{a}$ has a unique global solution $u(t)$ satisfying

$$
u(t) \in L_{\mathrm{loc}}^{\infty}\left(\mathbb{R}^{3} ; H^{0,1}\left(\mathbb{R}^{3}\right)\right) \cap L_{\mathrm{loc}}^{2}\left(\mathbb{R}^{3} ; H^{1,1}\left(\mathbb{R}^{3}\right)\right) \cap L_{\mathrm{loc}}^{2 \alpha+2}\left(\mathbb{R}^{3} ; L^{2 \alpha+2}\left(\mathbb{R}^{3}\right)\right) .
$$

Moreover, the solution is in $C^{0}\left(\mathbb{R}^{3} ; L^{2}\left(\mathbb{R}^{3}\right)\right)$ and depends continuously on the initial data.

\section{Proof of Theorem 0.1}

The rest of the paper is dedicated to the proof of Theorem 0.1 In general, the proof is structured in four steps. First, one defines a family of approximate systems $\left(\mathcal{S}_{a}^{n}\right)_{n \in \mathbb{N}}$ and show that this family has local in time smooth enough solutions $\left(u_{n}(t), p_{n}(t)\right)$. This can be achieved for instance by the classical Friedrich's method. Second, one proves uniform bounds for $\left(u_{n}(t), p_{n}(t)\right)$ on some fixed time interval $[0, T]$. Next, one shows that the sequence of solutions to $\left(\mathcal{S}_{a}^{n}\right)_{n \in \mathbb{N}}$ converges towards some solution of $\mathcal{S}_{a}$ with adequate properties. Eventually, one exhibits a stability kind estimate leading to the continuous dependence of the solutions on the initial data, in particular their uniqueness. We refer to any textbook of fluid mechanics for technical details of this procedure (see e.g. 1, 13]). To shorten the presentation, we will only present the necessary uniform bounds by performing formal calculation using system $\mathcal{S}_{a}$ instead of $\left(\mathcal{S}_{a}^{n}\right)_{n \in \mathbb{N}}$ and we will briefly explain how to pass to the limit.

A priori Estimates. We start by looking for an $L^{2}$ uniform estimate for the velocity. For this purpose, we multiply the first equation of system $\mathcal{S}_{a}$ by $u$ and integrate1 over $\mathbb{R}^{3}$ to get

$$
\frac{1}{2} \frac{d}{d t}\|u(t)\|_{2}^{2}+\nu\left\|\nabla_{h} u(t)\right\|_{2}^{2}+a\|u(t)\|_{2 \alpha+2}^{2 \alpha+2}=0,
$$

thanks to the fact that $\int_{\mathbb{R}^{3}}(u \cdot \nabla) u \cdot u d x=0$. Now, we integrate this equality with respect to time

$$
\|u(t)\|_{2}^{2}+2 \nu \int_{0}^{t}\left\|\nabla_{h} u(\tau)\right\|_{2}^{2} d \tau+2 a \int_{0}^{t}\|u(t)\|_{2 \alpha+2}^{2 \alpha+2} d \tau=\left\|u_{0}\right\|_{2}^{2} .
$$

This shows that if $u_{0} \in L^{2}\left(\mathbb{R}^{3}\right)$, then for all $t \in[0, T]$, it holds

$$
u(t) \in L^{\infty}\left(\mathbb{R}^{3} ; L^{2}\left(\mathbb{R}^{3}\right)\right) \cap L_{\mathrm{loc}}^{2}\left(\mathbb{R}^{3} ; H^{1,0}\left(\mathbb{R}^{3}\right)\right) \cap L_{\text {loc }}^{2 \alpha+2}\left(\mathbb{R}^{3} ; L^{2 \alpha+2}\left(\mathbb{R}^{3}\right)\right) .
$$

Next, we multiply the first equation of $\mathcal{S}$ by $-\partial_{3}^{2} u$ and integrate ${ }^{1}$ over $\mathbb{R}^{3}$ to get

$$
\begin{aligned}
\frac{1}{2} \frac{d}{d t}\left\|\partial_{3} u(t)\right\|_{2}^{2}+\nu\left\|\nabla_{h} \partial_{3} u(t)\right\|_{2}^{2} & -\int_{\mathbb{R}^{3}}(u(t) \cdot \nabla) u(t) \cdot \partial_{3}^{2} u(t) d x \\
& -a \int_{\mathbb{R}^{3}}|u(t)|^{2 \alpha} u(t) \cdot \partial_{3}^{2} u(t) d x=0 .
\end{aligned}
$$

\footnotetext{
${ }^{1}$ This should be done on the smooth approximate solutions $u_{n}$.
} 
Now, we handle the nonlinear terms. On the one hand, an integration by parts leads clearly to the fact that

$$
\begin{aligned}
-\int_{\mathbb{R}^{3}}|u|^{2 \alpha} u \cdot \partial_{3}^{2} u d x & =\int_{\mathbb{R}^{3}}\left|\partial_{3} u\right|^{2}|u|^{2 \alpha} d x+2 \alpha \int_{\mathbb{R}^{3}}\left(u \cdot \partial_{3}\right)^{2}|u|^{2 \alpha-2} d x \\
& =(1+2 \alpha)\left\||u|^{\alpha} \partial_{3} u\right\|_{2}^{2} .
\end{aligned}
$$

On the other hand, using integration by parts, we can write

$$
\begin{aligned}
-\int_{\mathbb{R}^{3}}(u \cdot \nabla) u \cdot \partial_{3}^{2} u d x & =\sum_{k, l=1}^{3} \int_{\mathbb{R}^{3}} \partial_{3} u_{k} \partial_{k} u_{l} \partial_{3} u_{l} d x \\
& =\sum_{k=1}^{2} \sum_{l=1}^{3} \int_{\mathbb{R}^{3}} \partial_{3} u_{k} \partial_{k} u_{l} \partial_{3} u_{l} d x \\
& +\sum_{l=1}^{3} \int_{\mathbb{R}^{3}} \partial_{3} u_{3} \partial_{3} u_{l} \partial_{3} u_{l} d x:=\mathscr{T}_{1}+\mathscr{T}_{2} .
\end{aligned}
$$

Now, integrating again by parts, we obtain that

$$
\mathscr{T}_{1}=-\sum_{k=1}^{2} \sum_{l=1}^{3} \int_{\mathbb{R}^{3}}\left(u_{l} \partial_{3} u_{l} \partial_{k} \partial_{3} u_{k}+u_{l} \partial_{3} u_{k} \partial_{k} \partial_{3} u_{l}\right) d x
$$

Moreover, using the fact that $\nabla \cdot u=0$, we have $-\partial_{3} u_{3}=\operatorname{div}_{h} u_{h}$ where $u_{h} \delta\left(u_{1}, u_{2}\right)$. Thus

$$
\mathscr{T}_{2}=-\sum_{l=1}^{3} \int_{\mathbb{R}^{3}} \operatorname{div}_{h} u_{h} \partial_{3} u_{l} \partial_{3} u_{l} d x
$$

Next, using Hölder and Young inequality, it is rather easy to see that for all $f, g$ and $h$, we have for all $\alpha>1$ and $\epsilon_{0}, \epsilon_{1}>0$

$$
\begin{aligned}
\int_{\Omega} f g h d x \leq \int_{\Omega}|f||g|^{\frac{1}{\alpha}}|g|^{1-\frac{1}{\alpha}}|h| d x & \leq\left\||f||g|^{\frac{1}{\alpha}}\right\|_{2 \alpha}\left\||g|^{1-\frac{1}{\alpha}} \mid\right\|_{\frac{2 \alpha}{\alpha-1}}\|h\|_{2} \\
& \leq \frac{1}{4 \epsilon_{0}}\left\|f^{\alpha} g\right\|_{2}^{\frac{2}{\alpha}}\|g\|_{2}^{2\left(1-\frac{1}{\alpha}\right)}+\epsilon_{0}\|h\|_{2}^{2} \\
& \leq \frac{\epsilon_{1}}{4 \epsilon_{0}}\left\|f^{\alpha} g\right\|_{2}^{2}+\frac{\epsilon_{1}^{\frac{1}{1-\alpha}}}{4 \epsilon_{0}}\|g\|_{2}^{2}+\epsilon_{0}\|h\|_{2}^{2} .
\end{aligned}
$$

Eventually, applying this inequality with $f=u_{l}, g=\partial_{3} u_{l}, h=\partial_{k} \partial_{3} u_{k}$ for the first part of $\mathscr{T}_{1}, f=u_{l}, g=\partial_{3} u_{k}, h=\partial_{k} \partial_{3} u_{l}$ for the second part of $\mathscr{T}_{1}$ and proceeding equivalently for $\mathscr{T}_{2}$, we obtain the existence of a constant $\gamma>0$ independent of $\alpha$ such that

$$
\int_{\mathbb{R}^{3}}(u \cdot \nabla) u \cdot \partial_{3}^{2} u d x \leq \frac{\gamma \epsilon_{1}}{4 \epsilon_{0}}\left\||u|^{\alpha} \partial_{3} u\right\|_{2}^{2}+\frac{\gamma \epsilon_{1}^{\frac{1}{1-\alpha}}}{4 \epsilon_{0}}\left\|\partial_{3} u\right\|_{2}^{2}+\gamma \epsilon_{0}\left\|\nabla_{h} \partial_{3} u\right\|_{2}^{2} .
$$

The idea then is to tune $\epsilon_{0}$ and $\epsilon_{1}$ to compensate the first and third terms of the right hand side of (5) using (44) and the second term of the left hand side of (33). More precisely, setting $\epsilon_{0}=\frac{\nu}{2 \gamma}$ and $\epsilon_{1}=\frac{a \nu(1+4 \alpha)}{\gamma^{2}}$ and using (41) and (5), the equality (3) implies the existence of some $\eta>0$ such that

$$
\frac{d}{d t}\left\|\partial_{3} u(t)\right\|_{2}^{2}+\nu\left\|\nabla_{h} \partial_{3} u(t)\right\|_{2}^{2}+a\left\||u(t)|^{\alpha} \partial_{3} u(t)\right\|_{2}^{2} \leq \eta\left\|\partial_{3} u(t)\right\|_{2}^{2} .
$$


Thus, thanks to Gronwall's inequality, we obtain the following bound

$$
\left\|\partial_{3} u(t)\right\|_{2}^{2} \leq\left\|\partial_{3} u_{0}\right\|_{2}^{2} e^{\eta t}, \quad \text { for all } t \in[0, T] .
$$

In particular, we have

$$
\left\|\partial_{3} u(t)\right\|_{2}^{2}+\nu \int_{0}^{t}\left\|\nabla_{h} \partial_{3} u(\tau)\right\|_{2}^{2} d \tau+a \int_{0}^{t}\left\||u(\tau)|^{\alpha} \partial_{3} u(\tau)\right\|_{2}^{2} d \tau \leq\left(1+e^{\eta t}\right)\left\|\partial_{3} u_{0}\right\|_{2}^{2} .
$$

Thus, we infer

$$
u(t) \in L^{\infty}\left(\mathbb{R}^{3} ; H^{0,1}\right) \cap L^{2}\left(\mathbb{R}^{3} ; H^{1,1}\right) .
$$

Rigorously, these bounds hold for the approximate solutions constructed via the Friederich's regularization procedure. So, at this level, it remains only to pass to the limit in the sequence of solutions of $\left(\mathcal{S}_{a}^{n}\right)_{n \in \mathbb{N}}$. For that purpose, the main point to show is that

$$
\begin{aligned}
\partial_{t} u & \in L_{\mathrm{loc}}^{2}\left(\mathbb{R}^{3}, H^{-1}\left(\mathbb{R}^{3}\right)\right)+L_{\mathrm{loc}}^{1+\frac{1}{2 \alpha+1}}\left(\mathbb{R}^{3}, L^{1+\frac{1}{2 \alpha+1}}\left(\mathbb{R}^{3}\right)\right) \\
& =\left(L_{\mathrm{loc}}^{2}\left(\mathbb{R}^{3}, H^{1}\left(\mathbb{R}^{3}\right)\right) \cap L_{\mathrm{loc}}^{2 \alpha+2}\left(\mathbb{R}^{3}, L^{2 \alpha+2}\left(\mathbb{R}^{3}\right)\right)\right)^{\star},
\end{aligned}
$$

where the star stands for the dual symbol. Indeed, let us recall the Ladyžhenskaya inequality, which is a special case of the Gagliardo-Nirenberg-Sobolev inequality (see e.g. [9])

$$
\|\psi\|_{4} \leq \delta_{1}\|\psi\|_{2}^{\frac{1}{4}}\|\nabla \psi\|_{2}^{\frac{3}{4}}, \text { for all } \psi \in H_{0}^{1}\left(\mathbb{R}^{3}\right) .
$$

Therefore, using Hölder and Ladyžhenskaya inequalities, we have

$$
\|(u \cdot \nabla) u\|_{\frac{4}{3}} \leq\|u\|_{4}\|\nabla u\|_{2} \leq \delta_{1}\|u\|_{2}^{\frac{1}{4}}\|\nabla u\|_{2}^{\frac{7}{4}} \leq \delta_{1}^{8}\|u\|_{2}^{2}+\|\nabla u\|_{2}^{2} .
$$

Therefore $(u \cdot \nabla) u \in L_{\text {loc }}^{2}\left(\mathbb{R}^{3}, H^{-1}\left(\mathbb{R}^{3}\right)\right)$. Also, we have clearly

$$
\left\||u|^{2 \alpha} u\right\|_{1+\frac{1}{2 \alpha+1}}^{1+\frac{1}{2 \alpha+1}}=\|u\|_{2 \alpha+2}^{2 \alpha+2}, \quad \text { and } \quad(2 \alpha+2)^{-1}+(1+1 / 2 \alpha+1)^{-1}=1 .
$$

Thus, (7) holds thanks to (2), (6), (8) and (9). Recall that (7) is needed in order to get some compactness in time. The passage to the limit follows using using classical argument by combining Ascoli's theorem and the Cantor diagonal process.

Remark 0.2. It is rather standard to show that $u \in C^{0}\left(\mathbb{R}^{3} ; L^{2}\right)$ by using (6) and (77).

Uniqueness. Now, we show the continuous dependence of the solutions on the initial data, in particular their uniqueness. Let $u(t)$ and $v(t)$ be two solutions of system $\mathcal{S}$ in the class $u(t) \in L^{\infty}\left(\mathbb{R}^{3} ; H^{0,1}\right) \cap L^{2}\left(\mathbb{R}^{3} ; H^{1,1}\right)$. Let $w(t)=u(t)-v(t)$, then $w$ satisfies

$$
\left\{\begin{aligned}
\partial_{t} w-\nu \Delta_{h} w+(w \cdot \nabla) u+(v \cdot \nabla) w+a|u|^{2 \alpha} u-a|v|^{2 \alpha} v & =-\nabla\left(p_{u}-p_{v}\right), \\
\nabla \cdot w & =0, \\
u_{\left.\right|_{t=0}} & =u_{0}-v_{0},
\end{aligned}\right.
$$

We proceed as for the obtention of a priori estimates. Thanks to (7), the action of $\partial_{t} w$ on $w$ leads to

$$
\frac{1}{2} \frac{d}{d t}\|w\|_{2}^{2}+\nu\left\|\nabla_{h} w\right\|_{2}^{2}+\int_{\mathbb{R}^{3}}(w \cdot \nabla) u \cdot w d x+a \int_{\mathbb{R}^{3}}\left(|u|^{2 \alpha} u-|v|^{2 \alpha} v\right) w d x=0 .
$$


On the one hand

$$
\int_{\mathbb{R}^{3}}(w \cdot \nabla) u \cdot w d x=\sum_{k=1}^{2} \sum_{l=1}^{3} \int_{\mathbb{R}^{3}} w_{k} \partial_{k} u_{l} w_{l} d x+\sum_{l=1}^{3} \int_{\mathbb{R}^{3}} w_{3} \partial_{3} u_{l} w_{l} d x \delta \mathscr{I}_{1}+\mathscr{I}_{2} .
$$

Now, using Hölder inequality, it holds

$$
\begin{aligned}
\mathscr{I}_{1} & \leq \sum_{k=1}^{2} \sum_{l=1}^{3} \int_{\mathbb{R}}\left\|\partial_{k} u_{l}\right\|_{L_{h}^{2}}\left\|w_{k}\right\|_{L_{h}^{4}}\left\|w_{l}\right\|_{L_{h}^{4}} d x_{3} \\
& \leq \sum_{k=1}^{2} \sum_{l=1}^{3}\left\|\partial_{k} u_{l}\right\|_{L_{v}^{\infty}\left(L_{h}^{2}\right)}\left\|w_{k}\right\|_{L_{v}^{2}\left(L_{h}^{4}\right)}\left\|w_{l}\right\|_{L_{v}^{2}\left(L_{h}^{4}\right)} .
\end{aligned}
$$

Now, using the Sobolev embedding $\dot{H}_{h}^{\frac{1}{2}} \hookrightarrow L_{h}^{4}$ and interpolating $\dot{H}_{h}^{\frac{1}{2}}$ between $\dot{H}_{h}^{1}$ and $L_{h}^{2}$, we obtain clearly for all $\psi \in L_{v}^{2} \cap \dot{H}_{h}^{1}$

$$
\|\psi\|_{L_{v}^{2}\left(L_{h}^{4}\right)} \leq C\left\|\nabla_{h} \psi\right\|_{2}^{\frac{1}{2}}\|\psi\|_{2}^{\frac{1}{2}} .
$$

Also, we have

$$
\begin{aligned}
\left\|\psi\left(\cdot, x_{3}\right)\right\|_{L_{h}^{2}}^{2} & =\int_{-\infty}^{x_{3}} \frac{d}{d z}\left(\|\psi(\cdot, z)\|_{L_{h}^{2}}^{2}\right) d z \\
& =2 \int_{-\infty}^{x_{3}} \int_{\mathbb{R}^{2}} \psi\left(x_{h}, z\right) \partial_{z} \psi\left(x_{h}, z\right) d x_{h} d z \leq 2\|\psi\|_{2}\left\|\partial_{3} \psi\right\|_{2} .
\end{aligned}
$$

Therefore, using (10) with $\psi=w_{k}$ and $\psi=w_{l}$ and (11) with $\psi=\partial_{k} u_{l}$, we obtain thanks to Young's inequality

$\mathscr{I}_{1} \leq C\left\|\partial_{3} \nabla_{h} u\right\|_{2}^{\frac{1}{2}}\left\|\nabla_{h} u\right\|_{2}^{\frac{1}{2}}\left\|\nabla_{h} w\right\|_{2}\|w\|_{2} \leq \frac{\nu}{4}\left\|\nabla_{h} w\right\|_{2}^{2}+C\left(\left\|\partial_{3} \nabla_{h} u\right\|_{2}^{2}+\left\|\nabla_{h} u\right\|_{2}^{2}\right)\|w\|_{2}^{2}$.

Next, proceeding in the same way, we get

$$
\mathscr{I}_{2} \leq C\left\|w_{3}\right\|_{L_{v}^{\infty}\left(L_{h}^{2}\right)}\left\|\partial_{3} \nabla_{h} u\right\|_{2}^{\frac{1}{2}}\left\|\partial_{3} u\right\|_{2}^{\frac{1}{2}}\left\|\nabla_{h} w\right\|_{2}^{\frac{1}{2}}\|w\|_{2}^{\frac{1}{2}} .
$$

But, using the fact that $\nabla \cdot w=0$, thus $\operatorname{div}_{h} w_{h}=-\partial_{3} w_{3}$, we get

$$
\begin{aligned}
\left\|w_{3}\right\|_{L_{h}^{2}}^{2} & =2 \int_{-\infty}^{x_{3}} \int_{\mathbb{R}^{2}} w_{3}\left(x_{h}, z\right) \partial_{3} w_{3}\left(x_{h}, z\right) d x_{h} d z \\
& =-2 \int_{-\infty}^{x_{3}} \int_{\mathbb{R}^{2}} w_{3}\left(x_{h}, z\right) \operatorname{div}_{h} w_{h}\left(x_{h}, z\right) d x_{h} d z \\
& \leq 2\left\|\operatorname{div}_{h} w_{h}\right\|_{2}\left\|w_{3}\right\|_{2} .
\end{aligned}
$$

Hence

$$
\begin{aligned}
\mathscr{I}_{2} & \leq C\left\|\operatorname{div}_{h} w_{h}\right\|_{2}^{\frac{1}{2}}\left\|w_{3}\right\|_{2}^{\frac{1}{2}}\left\|\partial_{3} \nabla_{h} u\right\|_{2}^{\frac{1}{2}}\left\|\partial_{3} u\right\|_{2}^{\frac{1}{2}}\left\|\nabla_{h} w\right\|_{2}^{\frac{1}{2}}\|w\|_{2}^{\frac{1}{2}} \\
& \leq \frac{\nu}{4}\left\|\nabla_{h} w\right\|_{2}^{2}+C\left(\left\|\partial_{3} \nabla_{h} u\right\|_{2}^{2}+\left\|\partial_{3} u\right\|_{2}^{2}\right)\|w\|_{2}^{2}
\end{aligned}
$$

On the other hand, it is well known that there exists a nonnegative constant $\kappa=$ $\kappa(\alpha)$ such that

$$
0 \leq \kappa|u-v|^{2}(|u|+|v|)^{2 \alpha} \leq\left(|u|^{2 \alpha} u-|v|^{2 \alpha} v\right) \cdot(u-v) .
$$

Thus

$$
a \int_{\mathbb{R}^{3}}\left(|u|^{2 \alpha} u-|v|^{2 \alpha} v\right) w d x \geq a \kappa \int_{\mathbb{R}^{3}}(|u|+|v|)^{2 \alpha} w^{2} d x=a \kappa\left\|(|u|+|v|)^{\alpha} w\right\|_{2}^{2} .
$$


All in all, we have

$$
\begin{aligned}
\frac{d}{d t}\|w\|_{2}^{2}+\nu\left\|\nabla_{h} w\right\|_{2}^{2} & +2 a \kappa\left\|(|u|+|v|)^{\alpha} w\right\|_{2}^{2} \\
& \leq C\left(\left\|\partial_{3} \nabla_{h} u\right\|_{2}^{2}+\left\|\partial_{3} u\right\|_{2}^{2}+\left\|\nabla_{h} u\right\|_{2}^{2}\right)\|w\|_{2}^{2}
\end{aligned}
$$

Setting $\mathcal{L}(t):=C\left(\left\|\partial_{3} \nabla_{h} u\right\|_{2}^{2}+\left\|\partial_{3} u\right\|_{2}^{2}+\left\|\nabla_{h} u\right\|_{2}^{2}\right)$ and integrating the above inequality with respect to time, we obtain that for all $t \in[0, T]$

$$
\begin{aligned}
&\|w(t)\|_{2}^{2}+\nu \int_{0}^{t}\left\|\nabla_{h} w(\tau)\right\|_{2}^{2} d \tau+2 a \kappa \int_{0}^{t}\left\|(|u|+|v|)^{\alpha} w(\tau)\right\|_{2}^{2} d \tau \\
& \leq\left\|w_{0}\right\|_{2}^{2}+\int_{0}^{t} \mathcal{L}(\tau)\|w(\tau)\|_{2}^{2} d \tau
\end{aligned}
$$

Now, since $\partial_{3} u \in L_{\text {loc }}^{\infty}\left(\mathbb{R}^{3}, L^{2}\left(\mathbb{R}^{3}\right)\right), \partial_{3} \nabla_{h} u \in L_{\text {loc }}^{2}\left(\mathbb{R}^{3}, L^{2}\left(\mathbb{R}^{3}\right)\right)$ and $\nabla_{h} u \in L_{\text {loc }}^{2}\left(\mathbb{R}^{3}, L^{2}\left(\mathbb{R}^{3}\right)\right)$, it is clear that $\mathcal{L}(t) \in L_{\text {loc }}^{1}\left(\mathbb{R}^{3}\right)$. Therefore, Gronwall's Lemma applied to the inequality (12) leads to the uniqueness for all $\alpha>1$ and the proof is complete.

Acknowledgment: The research of Hakima Bessaih was partially supported by NSF grant DMS-1418838. The research of Saber Trabelsi was supported by the King Abdullah University of Science and Technology.

\section{REFERENCES}

[1] H. Bahouri, J.-Y. Chemin and R. Danchin, Fourier Analysis and Nonlinear Partial Differential Equations. Grundlehren der Mathematischen Wissenschaften [Fundamental Principles of Mathematical Sciences], 343. Springer, Heidelberg (2011).

[2] R. Bennacer, A. Tobbal and H. Beji, Convection naturelle Thermosolutale dans une Cavit Poreuse Anisotrope: Formulation de Darcy-Brinkman, Rev. Energ. Ren. Vol. 5, 1-21 (2002).

[3] X. Cai and Q. Jiu, Weak and strong solutions for the incompressible Navier-Stokes equations with damping, J. of Math. Ana. and App., 343 (2), 799-809 (2008).

[4] J.-Y. Chemin, B. Desjardins, I. Gallagher and E. Grenier,Fluids with anisotropic viscosity, M2AN, 34, pp: 315-335 (2000).

[5] J.-Y. Chemin, B. Desjardins, I. Gallagher and E. Grenier, Mathematical Geophysics. An Introduction to Rotating Fluids and the Navier-Stokes Equations, Oxford Lecture Series in Mathematics and its Applications, 32 (2006).

[6] E. Grenier and N. Masmoudi, Ekman layers of rotating fluid, the case of well prepared initial data, Comm. Partial Differential Equations, 22, no. 5-6, pp. 953-975 (1997).

[7] V. Kalantarov and S. Zelik, Smooth attractors for the Brinkman-Forchheimer equations with fast growing nonlinearities, Commun. Pure Appl. Anal., 11 (5), 2037-2054 (2012).

[8] D. Iftimie, A uniqueness result for the Navier-stokes equations with vanishing vertical viscosity, SIAM J. Math. Anal, Vol. 33, No. 6, pp. 1483-1493

[9] O.A. Ladyžhenskaya. The Mathematical Theory Of Viscous Incompressible Flow, Gordon and Breach Science Publishers, New York, (1969). Second English edi- tion, revised and enlarged. Mathematics and its Applications, Vol. 2.

[10] P. A. Markowich, E. S. Titi and S. Trabelsi Continuous data assimilation for the threedimensional Brinkman-Forchheimer-extended Darcy model, arXiv:1502.00964

[11] M. Paicu, em Équation anisotrope de Navier-Stokes dans des espaces critiques. Rev. Mat. Iberoamericana 21, no. 1, 179-235 (2005)

[12] J. Pedlosky, Geophysical Fluids Dynamics, Springer Verlag, New York (1987).

[13] R. Temam, Infinite Dimensional Dynamical Systems In Mchanics and Physics, SpringerVerlag, New York, (1997).

[14] J. Simon, Compact sets in the space $L^{p}(0, T ; B)$, Ann. Mat. Pura Appl. (4) 146, 65-96 (1987) 
(H. Bessaih) University of Wyoming, Department of Mathematics, Dept. 3036, 1000 East University Avenue, Laramie WY 82071, United States.

E-mail address: bessaih@uwyo.edu

(S. Trabelsi) Division of Math and Computer Sci. and Eng., King Abdullah University of Science and Technology, Thuwal 23955-6900, Saudi Arabia

E-mail address: saber.trabelsi@kaust.edu.sa

(H. Zorgati) Département de Mathématiques, Campus Universitaire, Université Tunis El Manar 2092, Tunisia.

E-mail address: hamdizorgati@yahoo.fr 